\title{
Estado nutricional de pacientes com esquizofrenia frequentadores do Centro de Atenção Psicossocial (CAPS) do Hospital de Clínicas de Porto Alegre
}

\author{
Nutritional status of patients with schizophrenia who attend the Psychosocial \\ Care Center (CAPS), Hospital de Clínicas de Porto Alegre
}

Karine Zortéa1,2, Lísia Rejane Guimarães, ${ }^{1,2}$, Clarissa Severino Gamaa ${ }^{1,2,3}$, Paulo Silva Belmonte-de-Abreu 1,2,3

\section{RESUMO}

Objetivo: Avaliar o estado nutricional de pacientes com esquizofrenia, atendidos por um programa de reabilitação social (CAPS). Métodos: Foi realizado um estudo transversal com 40 pacientes com diagnóstico de esquizofrenia, em uso de antipsicóticos, atendidos no CAPS do Hospital de Clínicas de Porto Alegre. Foram verificados medidas antropométricas (peso, estatura, circunferência abdominal, percentual de gordura corporal), pressão arterial e tabagismo. Resultados: A amostra constitui-se de 65\% de homens. A média de peso encontrada foi de 75,39 $\pm 15,73 \mathrm{~kg}$. O índice de massa corporal médio apresentou-se dentro dos parâmetros de sobrepeso $\left(26,76 \pm 4,78 \mathrm{~kg} / \mathrm{m}^{2}\right)$, sendo $55 \%$ dos pacientes com sobrepeso ou obesidade segundo a classificação da OMS/1998. A circunferência abdominal e o percentual de gordura corporal apresentaram-se elevados na maioria dos pacientes (62,5\% e 92,5\%, respectivamente). Adicionalmente, não houve associação significativa entre IMC e a classificação de antipsicóticos (típicos, atípicos, clozapina). Encontrou-se uma correlação entre o tempo de doença com o percentual de gordura $(r=0,39, p=0,033)$ e escolaridade com o peso $(r=0,362, p=0,046)$ e IMC $(r=0,372, p=0,039)$. Na regressão linear, $13 \%$ da variabilidade do percentual de gordura foi explicada pelo tempo de doença $\left(r^{2}=0,131, B=0,233, p\right.$ $=0,049) ; 13 \%$ da variação do peso foi explicada pela escolaridade $\left(r^{2}=0,131, B=1,415, p=\right.$ $0,046)$ e $13,8 \%$ da variação do IMC foi explicada pela escolaridade $\left(r^{2}=0,138, B=0,411, p=\right.$ 0,039). Conclusão: Os pacientes apresentaram níveis aumentados de circunferência abdominal, percentual de gordura corporal e peso. Aparentemente, o ganho de peso ocorre em todos os pacientes expostos a antipsicóticos, independentemente do tipo de medicação e de resposta clínica, e a qualquer momento ao longo da evolução da doença. Sugere-se que, adicionalmente, a avaliação dos hábitos alimentares e o acompanhamento nutricional desses pacientes, a detecção precoce de alterações associadas à exposição aos antipsicóticos,

\section{Palavras-chave}

Esquizofrenia, sobrepeso, obesidade, nutrição. em especial a obesidade, e o registro de mudanças ao longo do curso da doença e perante exposição a diferentes apresentações, tipos e doses de antipsicóticos sejam essenciais para o entendimento mais preciso do ganho de peso.
1 Hospital de Clínicas de Porto Alegre, Serviço de Psiquiatria, Laboratório de Psiquiatria Molecular.

2 Universidade Federal do Rio Grande do Sul (UFRGS), Programa de Pós-graduação em Ciências Médicas, Psiquiatria.

3 Instituto Nacional de Ciência e Tecnologia (INCT) Transnacional em Medicina.

Endereço para correspondência: Karine Zortéa

Av. Protásio Alves, 7157/203A, Petrópolis - 91310-003 - Porto Alegre, RS

Telefones: +55 (51) 8429-6901/+55 (51) 2101-8846

E-mail: karine.personaldiet@gmail.com 


\section{Keywords}

Schizophrenia, overweight, obesity, nutrition.

\section{ABSTRACT}

Objective: To evaluate the nutritional status of patients with schizophrenia attended in a social rehabilitation program (CAPS). Methods: Cross-sectional study with 40 patients diagnosed with schizophrenia, in antipsychotic use, attended in CAPS of the Hospital de Clínicas de Porto Alegre. Anthropometric measures were verified (weight, height, waist circumference, body fat percentage), blood pressure and cigarette smoking. Results: The sample consisted of $65 \%$ of men. Mean of weight was $75,39 \pm 15,73 \mathrm{~kg}$ and mean body mass index was within the parameters of overweight $\left(26,76 \pm 4,78 \mathrm{~kg} / \mathrm{m}^{2}\right), 55 \%$ of the patients with overweight or obesity according to the OMS/1998 criteria. Waist circumference and body fat percentage were high in most patients $162.5 \%$ and $92.5 \%$, respectively). In addition, there was no significant association between BMI and antipsychotics drugs (typic and atypic, clozapine). We found a correlation between duration of disease with body fat percentage $(r=0.39, p=0.033)$ and education correlated with weight $(r=0.362, p=0.046)$ and $B M I(r=0.372, p=0.039)$. In linear regression 13\% of the variability in fat percentage was explained by disease duration $\left(r^{2}=0.131, B=0.233, p=0.049\right)$, $13 \%$ of weight variation was explained by education $\left(r^{2}=0.131, B=1.415, p=0.046\right)$ and $13.8 \%$ of the variation in $B M I$ was explained by education level $\left(r^{2}=0.138, B=0.411, p=0.039\right)$. Conclusion: Patients showed increased levels of waist circumference, body fat percentage and overweight. Apparently, the weight gain occurs in all patients exposed to antipsychotics, independent from type of drug and clinical response, and at any moment along illness evolution. It is suggested that in addition to food habits an nutritional assessment and follow-up, the clinician should take notes about early changes along the course of illness, changes of type and dose of drugs.

\section{INTRODUÇÃO}

O uso de medicamentos antipsicóticos representa um importante componente no tratamento clínico de pacientes com esquizofrenia, porém tem sido associado a alterações metabólicas. O uso de antipsicóticos atípicos, com exceção da ziprasidona, é associado a obesidade, diabetes, dislipidemia, síndrome metabólica e elevada mortalidade ${ }^{1,2}$.

No Brasil, o uso de antipsicóticos atípicos aumentou muito nos últimos anos. Por isso, o impacto dos distúrbios metabólicos se tornou uma questão urgente, tanto na prática clínica diária como no sistema público de saúde, visto que alguns desses medicamentos fazem parte de um programa de alto custo do Ministério da Saúde. Outra peculiaridade do cenário nacional é o fato de a prática psiquiátrica na rede de atendimento primário preocupar-se, quase exclusivamente, com a remissão dos sintomas psicóticos, deixando de lado outros aspectos importantes do tratamento, como a segurança cardiovascular, sintomas negativos e distúrbios metabólicos, que acabam até mesmo aumentando a morbidade clínica e piorando a qualidade de vida dos pacientes com esquizofrenia ${ }^{3}$.

Além dos efeitos colaterais das medicações antipsicóticas, o estilo de vida sedentário e as escolhas dietéticas inadequadas contribuem para maior risco de obesidade nesses pacientes, quando comparados a outros indivíduos'. Nos últimos anos, a alta prevalência de sobrepeso e obesidade nos indivíduos com esquizofrenia tem sido bem descrita',2,4. Um estudo demonstrou que o índice de massa corporal (IMC) e o
LDL-colesterol estavam aumentados em pacientes que usavam maiores doses de clozapina em relação aos que usavam doses menores².

Adicionalmente, existe uma relação entre o aumento da circunferência abdominal e a baixa qualidade de vida nesses pacientes ${ }^{4,5}$. O IMC elevado e o ganho de peso também foram identificados como fatores associados a uma baixa qualidade de vida tanto em pacientes esquizofrênicos quanto na população geral ${ }^{5}$. Sua comprovada baixa qualidade de vida está relacionada com distúrbios alimentares, como a obesidade, e alterações metabólicas, como estresse oxidativo, de modo que esses pacientes merecem maior atenção no manejo e intervenção no controle do peso $0^{4,5}$.

Surge, então, o interesse de se avaliar o estado nutricional de pacientes com esquizofrenia que são atendidos por um programa de reabilitação social.

\section{MÉTODOS}

Trata-se de um estudo transversal no qual a população estudada compreendeu 40 indivíduos com idade superior a 18 anos. O diagnóstico de esquizofrenia foi realizado de acordo com critérios do DSM-IV e CID-10. Todos utilizavam antipsicóticos, no mínimo há três meses, e eram frequentadores do Centro de Atenção Psicossocial (CAPS) do Hospital de Clínicas de Porto Alegre (HCPA) no período de fevereiro a março de 2009. 
A coleta de dados foi realizada por meio de entrevista com os pacientes que aceitaram participar da pesquisa, que responderam oralmente a um questionário de anamnese e tiveram seus dados antropométricos (peso, estatura, circunferência abdominal e percentual de gordura) e pressão arterial medidos. A avaliação antropométrica foi realizada por nutricionistas da equipe. Com a utilização desses dados, foi possível realizar a classificação do estado nutricional dos pacientes. Os pacientes foram questionados sobre o tabagismo e sobre o número de cigarros consumidos por dia.

Para verificar o peso corporal, os indivíduos deveriam estar descalços, vestindo roupas leves. Foi utilizada uma balança digital antropométrica, da marca Filizola ${ }^{\circledR}$, devidamente aferida, e a estatura foi verificada com o auxílio de um estadiômetro afixado na parede, com variação de $0,1 \mathrm{~cm}$, onde foram posicionados em pé, descalços, com os calcanhares juntos, costas retas e os braços estendidos ao longo do corpo. Por meio da razão peso em kg/altura ${ }^{2}$ em metros, foi possível classificá-los quanto ao IMC em: baixo peso $\left(<18,49 \mathrm{~kg} / \mathrm{m}^{2}\right)$, eutrofia $\left(18,5-24,99 \mathrm{~kg} / \mathrm{m}^{2}\right)$, sobrepeso $\left(25-29,99 \mathrm{~kg} / \mathrm{m}^{2}\right)$ e obesidade $\left(\geq 30 \mathrm{~kg} / \mathrm{m}^{2}\right)$, de acordo com FAO/OMS (1998)6.

A pressão arterial foi aferida com o paciente sentado, após 5 minutos de repouso, conforme procedimentospadrão, sendo classificada como pressão sanguínea elevada quando $\geq 130 / 85 \mathrm{mmHg}^{7}$.

A circunferência abdominal foi medida na metade da distância entre a crista ilíaca e o rebordo costal inferior ${ }^{8}$, com auxílio de fita métrica inelástica, tendo como unidade de referência de circunferência abdominal elevada: para homens acima de $94 \mathrm{~cm}$ e para mulheres acima de $80 \mathrm{~cm}^{9}$.

O percentual de gordura foi obtido por meio de bioimpedância elétrica da marca Omron BF $300^{\circledR}$, classificado como: acima da média quando $\geq 16 \%$ para homens e $\geq 24 \%$ para mulheres; valores superiores a $25 \%$ para homens e $32 \%$ para mulheres representam risco para doenças associadas à obesidade ${ }^{10}$.

Para classificação dos sintomas psiquiátricos, foi aplicada a escala BPRS (Brief Psychiatric Rating Scale), traduzida e adaptada para o português por Zuardi et al. ${ }^{11}$. A escala possui 18 itens, em que considera tanto medidas observacionais quanto a partir de entrevista. Foi aplicada por um psiquiatra da equipe, devidamente habilitado para tal. Posteriormente, foi subdividida em sintomas positivos e sintomas negativos.

Os antipsicóticos foram divididos em três categorias (típicos, atípicos e clozapina).

Este estudo utilizou dados parciais do projeto "Funcionalidade e estresse oxidativo em pacientes esquizofrênicos com e sem obesidade", que tem aprovação do Comitê de Ética e Pesquisa em Saúde do HCPA (08-436). Todos os pacientes assinaram o Termo de Consentimento Livre e Esclarecido.

Os dados coletados estão apresentados de forma descritiva, com média e desvio-padrão (paramétricos), frequências ou mediana e intervalo de 95\% de confiança (não paramétri- cos). As associações entre dados paramétricos foram avaliadas por meio de coeficiente de correlação de Pearson e os dados não paramétricos, por meio do coeficiente de correlação de Spearman, e posteriormente foi utilizada análise de regressão linear. Variáveis categóricas foram analisadas com o teste de qui-quadrado. Para análise estatística, foi utilizado o programa SPSS 17.0. Um $p<0,05$ foi considerado significante.

\section{RESULTADOS}

Os dados descritivos da amostra estão apresentados na tabela 1. A idade média dos indivíduos foi de 38,7 \pm 9,41 anos, sendo $65 \%$ do sexo masculino. A média de peso encontrada foi de 75,39 $\pm 15,73 \mathrm{~kg}$ e o IMC médio encontrou-se dentro dos parâmetros de sobrepeso $\left(26,76 \pm 4,78 \mathrm{~kg} / \mathrm{m}^{2}\right)$. Segundo a classificação da OMS/19986,45\% dos pacientes encontramse eutróficos e $55 \%$, com sobrepeso/obesidade (30\% com sobrepeso; $25 \%$ com obesidade).

Tabela 1. Dados descritivos da amostra de 40 pacientes com diagnóstico F.20 - Esquizofrenia

\begin{tabular}{ll}
\hline Caracteristicas & Valor(DP) \\
\hline Idade (anos)* & $38,7 \pm 9,41$ \\
Escolaridade (anos estudados)* & $9,03 \pm 3,41$ \\
Tempo de doença (anos)** & $17(6,5-23,5)$ \\
Idade de início da doença (anos)* & $20,48 \pm 7,21$ \\
Gênero masculino*** (\%) & 65,0 \\
Uso de tabaco*** (\%) & 40,0 \\
Uso de drogas ilíititas** (\%) & 13,5 \\
Dados antropométricos* & \\
Peso (kg) & $75,39 \pm 15,74$ \\
IMC (kg/m $\left.{ }^{2}\right)$ & $26,76 \pm 4,78$ \\
Gordura corporal (\%) & $29,30 \pm 7,57$ \\
Circunferência abdominal (cm) & $94,75 \pm 13,2$ \\
Tipo de antipsicótico*** & \\
Primeira geração (\%) & 26,3 \\
Segunda geração (\%) & 28,9 \\
Clozapina (\%) & 44,7 \\
Psicopatologia** & \\
BPRS total & $16(10-29,5)$ \\
BPRS sintomas positivos & $4(0-9)$ \\
BPRS sintomas negativos & $5(2-7,5)$ \\
\hline
\end{tabular}

* Média \pm DP; ** Mediana (95\% IC); *** Frequência (\%); IMC: índice de massa corporal.

As medidas de circunferência abdominal e percentual de gordura corporal apresentaram-se elevadas na maioria dos pacientes (62,5\% e 92,5\%, respectivamente). Encontrou-se pressão arterial normal em $70 \%$ dos pacientes, $20 \%$ apresentaram níveis elevados e 10\%, níveis limítrofes. 
O tabagismo esteve presente em 40\% da amostra, e a maioria fumava uma (20\%) ou duas (12,5\%) carteiras de cigarro ao dia.

Não foi evidenciada correlação entre dados antropométricos em relação a sintomas, idade de início da doença, dose e tempo de uso da medicação.

O tempo de doença se correlacionou com o percentual de gordura ( $r=0,39, p=0,033)$. A escolaridade correlacionou-se com o peso ( $r=0,362$, $p=0,046)$ e IMC ( $r=0,372$, $p=0,039)$. $O$ estudo de regressão linear estimou que $13 \%$ da variabilidade do percentual de gordura foi explicada pelo tempo de doença $\left(r^{2}=0,131, B=0,233, p=0,049\right)$, com aumento anual de 0,23\% de peso. Adicionalmente, 13\% da variação do peso foi explicada pela escolaridade $\left(r^{2}=0,131\right.$, $B=1,415$, $p=0,046)$, e a cada ano estudado o peso aumentou em média $1,41 \mathrm{~kg}$. A regressão da escolaridade e IMC mostrou que $13,8 \%$ da variação do IMC foi explicada pela escolaridade $\left(r^{2}=0,138, B=0,411, p=0,039\right)$, e a cada ano estudado o IMC aumentou 0,411 kg/m².

Não houve associação entre a classificação do IMC e as categorias de antipsicóticos.

\section{DISCUSSÃO}

Os pacientes apresentaram níveis aumentados de circunferência abdominal, percentual de gordura corporal, e a maioria encontra-se com excesso de peso. Observaram-se 30\% dos pacientes com pressão arterial em valores superiores aos preconizados. Esses níveis foram aumentados em todos os subgrupos de pacientes (refratários, usuários de diferentes antipsicóticos, idade de início de doença, dose e tempo de uso de medicação).

Atualmente, o sobrepeso e a obesidade são comorbidades comuns entre esses pacientes, e tem sido demonstrada a presença de IMC significativamente maior em relação aos pacientes psiquiátricos sem o diagnóstico de esquizofrenia e à população de maneira geral. No Brasil, dados do Ministério da Saúde mostram prevalência de 32\% para o sobrepeso e $8 \%$ para a obesidade 3 . Neste estudo, encontraram-se $30 \%$ de pacientes com sobrepeso e $25 \%$ com obesidade, o que evidencia os níveis aumentados de obesidade nessa população. É importante ressaltar que esses indicadores são fatores de risco tanto para doenças cardiovasculares quanto para o desenvolvimento de síndrome metabólica³.

Além disso, estudos prévios sugerem que o excesso de peso pode reduzir a autoestima, possibilitando o abandono do tratamento psiquiátrico ${ }^{12}$. Pacientes tratados com antipsicótico, além de apresentarem significante aumento de peso, podem desenvolver anormalidades lipídicas, como a elevação dos níveis de LDL-colesterol e triglicerídeos. Sendo assim, necessitam de monitoramento frequente desses ní- veis plasmáticos e intervenção nutricional precoce, visando aperfeiçoar o tratamento ${ }^{2,13}$.

Também se observa alta frequência de tabagismo, com elevado consumo diário de cigarros. O tabagismo, o uso do álcool, a dieta inadequada e o sedentarismo aumentam a mortalidade na população em geral e necessitam de atenção também nesse grupo de pacientes ${ }^{3}$.

Tanto o estilo de vida quanto o tratamento com antipsicóticos podem contribuir para causar ou agravar alterações metabólicas nos indivíduos com transtornos psiquiátricos ${ }^{3,13}$. Diversas evidências sugerem que, na vigência de ganho de peso ou alterações metabólicas, o psiquiatra deve ponderar adequadamente a escolha da medicação, sempre levando em conta a fase de tratamento da doença e a eficácia obtida em relação aos efeitos colaterais indesejados ${ }^{3}$. Deve-se dar atenção ao papel da intervenção sobre fatores de risco de doenças cardiovasculares. Atitudes simples como pesar os pacientes, solicitar que façam um diário alimentar e encaminhá-los a um nutricionista se mostram como intervenções muito úteis ${ }^{13}$. Pesquisas preliminares com grupos de apoio para o controle do peso associado ao tratamento, especificamente elaborado para pacientes com esquizofrenia, têm demonstrado resultados promissores ${ }^{14}$.

$\mathrm{Na}$ amostra estudada, a escolaridade correlacionou-se com o peso e o IMC, o que demonstra que os pacientes com mais anos estudados apresentaram também maior peso. O tempo de doença se correlacionou com o percentual de gordura, o que pode ser explicado pelo maior tempo de uso de medicações antipsicóticas, apesar de não ter sido evidenciada correlação entre dados antropométricos em relação a dose e tempo de uso da medicação, bem como em relação a sintomas e idade de início da doença.

O estado nutricional dos pacientes, classificado de acordo com o IMC, não demonstrou associação com as categorias de antipsicóticos (típicos, atípicos e clozapina).

Aparentemente, o ganho de peso ocorre em todos os pacientes expostos a antipsicóticos, independentemente do tipo de medicação e de resposta clínica, e ocorre a qualquer momento ao longo da evolução da doença. Entretanto, não é possível afirmar sem antes realizar uma adequada revisão do período de ganho de peso, histórico de uso de diferentes medicações ao longo da doença e de comparação com pacientes não usuários de antipsicóticos.

Este estudo apresenta algumas limitações: primeiramente o desenho transversal, não randomizado, que não permitiu identificar causa e efeito, e particularmente por não se ter os dados de peso antes do início do tratamento medicamentoso; em segundo lugar, por ser um estudo que envolve pacientes crônicos, medicados em longo prazo, seus dados não podem ser expandidos para pacientes em primeiro episódio. Novos estudos são necessários para avaliar as características metabólicas dos pacientes no estágio inicial da doença, bem como estudos longitudinais, caso-controle, para esclarecer 
as mudanças que ocorrem no decorrer dessa patologia e compará-las com indivíduos saudáveis.

\section{CONCLUSÃO}

Grande parte dos pacientes apresentou excesso de peso, circunferência abdominal e percentual de gordura corporal elevados. Esses são importantes fatores de risco para doenças cardiovasculares e síndrome metabólica. Portanto, além da clínica médica, psiquiátrica e psicológica, sugere-se que, adicionalmente, se façam a avaliação dos hábitos alimentares e o acompanhamento nutricional desses pacientes, para detecção precoce de alterações associadas à exposição aos antipsicóticos, em especial a obesidade, o registro de mudanças ao longo do curso da doença e da exposição a diferentes apresentações, tipos e doses de antipsicóticos, para entendimento mais preciso do ganho de peso, visando à prevenção de doenças associadas à obesidade.

\section{CONFLITO DE INTERESSE}

Os autores declaram não existir conflito de interesse.

\section{REFERÊNCIAS}

1. Leitão-Azevedo CL, Guimarães LR, Lobato MI, et al. Ganho de peso e alterações metabólicas em esquizofrenia. Rev Psiquiatr Clin. 2007;34(2):184-8.
2. Zortéa K, Bock PM, Moreno DB, Belmonte de Abreu PS. Avaliação antropométrica e bioquímica em pacientes com esquizofrenia usuários de clozapina. Rev Nutr. 2009;22(5):697-705

3. Elkis H, Gama C, Suplicy H, Tambascia M, Bressan R, Lyra R, et al. Consenso Brasileiro sobre antipsicóticos de segunda geração e distúrbios metabólicos. Rev Bras Psiquiatr. 2008;30(1):77-85.

4. Faulkner G, Cohn T, Remington G, Irving H. Body mass index, waist circumference and quality of life in individuals with schizophrenia. Schizophr Res. 2007;90(1-3):174-8.

5. Cardoso CS, Caiaffa WT, Bandeira M, Siqueira AL, Abreu MA, Fonseca J0. Factors associated with a low quality of life in schizophrenia. Cad Saude Publica. 2005;21:1338-48.

6. FA0/OMS. Physical status: the use and interpretation of anthropometry. Genebra; 1998.

7. Executive Summary of the Third Report of the National Cholesterol Education Program (NCEP) Expert Panel on Detection, Evaluation, and Treatment of High Blood Cholesterol in Adults (Adult Treatment Panel III). JAMA. 2001;285(19):2486-97.

8. Brasil. I Diretriz Brasileira de Diagnóstico e Tratamento da Síndrome Metabólica. Arq Bras Cardiol. 2005;84(1):3-28.

9. Brasil. IV Diretriz Brasileira sobre as Dislipidemias e Prevenção da Aterosclerose do Departamento de Aterosclerose da Sociedade Brasileira de Cardiologia. Arq Bras Cardiol. 2007;88(1)2-19.

10. Lohman TG. Advances in body composition assessment. Current issues in exercise science series. Monografh n. 3. Champaign, IL: Human Kinetics; 1992.

11. Zuardi AW, Loureiro SR, Rodrigues CRC, Correia AJ, Glock SS. Estudo da estrutura fatorial, fidedignidade e validade da tradução e adaptação para o português da Escala de Avaliação Psiquiátrica Breve (BPRS) modificada. In: Gorenstein C, Andrade LHSG, Zuardi AW. Escalas de Avaliação em Psiquiatria e Psicofarmacologia. São Paulo: Lemos Editorial; 2000.

12. De Hert M, Peuskens B, Winkel RV, Kalnicka D, Hanssens L, Eyck DV, et al. Body weight and self-esteem in patients with schizophrenia evaluated with B-WISE ${ }^{\circledR}$. Schizophr Res. 2006:88(1-3):222-6.

13. Cerqueira Filho EA, Arandas FS, Oliveira IR, Sena EP. Dislipidemias e antipsicóticos atípicos. J Bras Psiquiatr. 2006;55(4):296-307.

14. Wirshing DA. Schizophrenia and obesity: impact of antipsychotic medications. J Clin Psychiatry. 2004;65(18):13-26. 